\title{
Cascaded versus noncascaded models of lexical and semantic processing: The turple effect
}

\author{
KENNETH I. FORSTER and JO HECTOR \\ University of Arizona, Tucson, Arizona
}

\begin{abstract}
The density of the orthographic neighborhood surrounding an item has been shown to have an inhibitory effect for nonwords in a lexical decision experiment. Four experiments are reported investigating whether a similar pattern holds for a semantic categorization task (animal vs. non-animal). In the first experiment, no effects of neighborhood density were found for nonexemplars, whether they were words or nonwords. The absence of any inhibitory effect for nonwords implies that close orthographic neighbors are ignored. However, the second experiment showed that if the nonword has a neighbor that is an animal name (e.g., turple), an interference effect is observed, implying that neighbors do exert an effect if they have the right semantic properties. The same items showed no additional interference in lexical decision. These results suggest the involvement of semantic properties very early in the processing cycle. A cascaded processing system monitoring activation in semantic features can explain these results, but cannot explain the frequency effect observed for nonexemplar words or the fact that variation in density is irrelevant when one of the neighbors is an exemplar. It is argued that existing models of semantic categorization must be extended to accommodate these results.
\end{abstract}

In recent years the study of visual word recognition has focused largely on the way in which the orthographic neighborhood of a word influences its perception. A word is said to be located in a dense neighborhood if it has many neighbors, where a neighbor is defined as a word spelled the same way except for one letter (Coltheart, Davelaar, Jonasson, \& Besner, 1977). Intuitively, one would expect the recognition of a word in a dense neighborhood to require a finer discrimination than a word in a sparse neighborhood, and therefore high-density words should be more difficult to identify, especially if the neighbors are higher in frequency. Similarly, in a lexical decision task, deciding that an item is a nonword should be more difficult when it closely resembles a number of actual words. Surprisingly, the evidence from lexical decision experiments is highly mixed, at least for words. Although some investigators have reported inhibitory effects of a higher frequency neighbor for words (Grainger, O'Regan, Jacobs, \& Segui, 1989; Grainger \& Segui, 1990), attempts to replicate this finding have not all met with success, at least for English (Forster \& Shen, 1996; Sears, Hino, \& Lupker, 1995, but see Perea $\&$ Pollatsek, 1998). Other investigators have reported a fa-

This research was supported in part by National Multipurpose Research and Training Grant 1P60 DC01409 from the National Institute on Deafness and Other Communication Disorders to the National Center for Neurogenic Communications Disorders at the University of Arizona. The authors wish to express their thanks to Max Coltheart and Colin Davis for extensive discussion of theoretical issues. Correspondence should be addressed to K. I. Forster, Department of Psychology, University of Arizona, Tucson, AZ 85721 (e-mail: kforster@u.arizona. edu). cilitatory effect of the number of neighbors for lowfrequency words, but no effect for high-frequency words (Andrews, 1989, 1992, 1997; Forster \& Shen, 1996; Sears et al., 1995), and others have suggested that the number of neighbors has no effect at all for words (Coltheart et al., 1977). Despite this uncertainty about the effect for words, there is wide agreement that a dense neighborhood has an inhibitory effect on lexical decision times for nonwords (Andrews, 1989, 1992; Coltheart et al., 1977; Forster \& Shen, 1996; Johnson \& Pugh, 1994; Sears et al., 1995). We refer to this effect as the nonword $N$ effect, where $N$ represents the number of neighbors.

In an effort to encompass these contradictory findings, Grainger and Jacobs (1996) developed the multiple readout model (MROM), a computational model, which proposed that decisions about words could be based on activation at different levels of processing. In a lexical decision task, decisions could be based on activation in an individual word unit or could be based on an index of global activation obtained by summing activation levels over all word units. Words produce higher global activation levels than do nonwords, and hence it is possible to make a reasonably accurate guess in a lexical decision task without waiting for a word unit to reach criterion. In such a model, neighbors could have opposite effects, depending on which index was used. An inhibitory effect of a neighbor might be expected when the response is based on activation in an individual word unit, due to lateral inhibition between word units. On the other hand, a facilitatory effect would be expected when the response is based on global activation. The reason for this is that a word will produce activation in the units of all its neighbors, leading to higher 
global activation levels, which means that the criterion level for a "yes" response will be reached faster. But for nonwords, a relatively high level of global activationleads to problems. Normally, a "no" decision is made when there has been insufficient activation for a specified period of time (i.e., a deadline model). However, for nonwords with several neighbors, the activation level is not high enough to warrant a "yes" decision, nor is it low enough to warrant a "no" decision. The solution to this dilemma, as originally suggested by Coltheart et al. (1977), is to adopt a "wait and see" strategy, which involves lengthening the deadline for making a "no" decision (see also discussion of the dual-route cascaded [DRC] model of Coltheart, Rastle, Perry, Langdon, \& Ziegler, 2001). Hence the presence of neighbors lengthens decision times for nonwords.

This explanation in terms of global activation works well for the lexical decision task, but it is not clear that it applies to other tasks. For example, in a semantic categorization task, the word must be classified according to some semantic criterion (e.g., whether it is the name of an animal). In this case, it is irrelevant whether the global activation level is high or low. Decisions now must be based on activation at the semantic level. This activation is generated by activation in a word unit, and hence one might expect the facilitatory effect of neighbors to disappear, whereas the inhibitory effect produced by lateral inhibition should remain. However, previous research using semantic categorization to investigate $N$ effects has produced mixed results. Forster and Shen (1996) reported no effect of $N$ for nonexemplar words in an animal categorization experiment, but Sears, Lupker, and Hino (1999) found a facilitatory effect, and Carreiras, Perea, and Grainger (1997) found an inhibitory effect in the presence of a higher frequency neighbor and a facilitatory effect when there was no higher frequency neighbor. Clearly, it will be difficult to reconcile these findings, which may say something about the difficulty of comparing responses to different sets of words.

None of these studies asked what would happen to the effect of $N$ on nonwords in such an experiment, and because there is far more agreement about the nonword $N$ effect, it may be that an examination of this effect will provide a useful perspective. The nonword $N$ effect is especially relevant for models of lexical access that assume that the input generates a set of candidates based on form similarity that must then be subjected to a verification processfor example, the activation-verification model of Paap, Newsome, McDonald, and Schvaneveldt (1982), the entryopening model (Forster, 1999; Forster \& Davis, 1984; Forster \& Veres, 1998), and the short-list model (Norris, 1986). This verification process involves comparing the spelling of each candidate with the input letter sequence. In a lexical decision task, a "yes" decision is made if one of these candidates is found to match the input. However, a "no" decision must be delayed until all candidates have been eliminated. In models that explicitly assume that candidates are evaluated serially (e.g., the activation-verification model or the entry-opening model), it is clear that $N$ must have an inhibitory effect on "no" decisions because the more candidates there are, the longer it will take to eliminate all of them. Whether this verification process has any implications for "yes" decisions depends on other assumptions. For example, in the activation-verification model, candidates are verified in an order determined by frequency of occurrence, and hence the existence of a higher frequency neighbor should delay the verification of the correct candidate. However, in the entry-opening model (Forster, 1999), it is specifically assumed that candidates receiving a high goodness-of-fit score are evaluated as soon as they are detected (interrupting the evaluation of any other candidates), and hence a higher frequency neighbor should have no effect.

The prediction of an inhibitory nonword $N$ effect can be extended to the semantic categorization task in the following way. The assumption underlying the verification process is that the initial stage of processing is noisy, and hence any one of the candidates might turn out to be the matching entry. If such a match is found, its semantic properties must then be compared with the task category. If the matching candidate has the right semantic properties, a "yes" decision can be made. If it does not have the right properties, it is rejected, and the next candidate is considered. Hence a "no" decision must again be delayed until all candidates have been verified. However, there is an important qualification to this argument. This prediction depends on the assumption that semantic properties are not available until there has been resolution at the level of form (i.e., one of the candidates has been successfully verified). This means that there is no way to evaluate the semantic properties of candidates prior to verification. We shall refer to such an assumption as a "form-first" assumption. This is a common assumption of modular "stage" models, in which it is assumed that processing at each level must be completed before processing at the next higher level can commence. However, such an assumption is not typical of activation models. For example, in the DRC model of Coltheart et al. (2001), activation is cascaded, so activation is passed from the word level to the semantic level with no waiting for resolution at the level of form. This is also true of connectionistmodels of word recognition (e.g., the triangle model of Plaut, McClelland, Seidenberg, \& Patterson, 1996). Under these conditions, a more efficient method for making semantic categorizations might be to simply monitor activation in the appropriate semantic feature units. In this case, activation levels in the word units for neighbors would be irrelevant because the decision procedure is tuned to activity at the semantic level.

Just such a model has been proposed by Carreiras et al. (1997) as an extension to the MROM model (Grainger \& Jacobs, 1996). In this model, it is assumed that in an animal categorization experiment, decisions are based on the activation level in an "animalness" feature. A "yes" decision is generated if the level of activation in this feature reaches criterion; a "no" decision is generated when a deadline expires. However, there is no reason to extend this 
deadline on account of neighbors, because the only relevant property of the word is the semantic activation it produces. Thus, in this model, decisions for nonwords (and nonexemplar words) are generated when a deadline expires, and hence the number of neighbors would be irrelevant to the decision.

It appears, then, that the question of whether there is a nonword $N$ effect in semantic categorization is highly relevant to core assumptions about cognitive architecture. Form-first models predict that the nonword $N$ effect should be present, whereas a cascaded feature monitoring model does not. The present series of experiments was designed to decide between these alternatives.

\section{EXPERIMENT 1}

The primary purpose of this experiment was to test whether high- $N$ nonwords take longer to classify in a semantic categorization task than low- $N$ nonwords. The task was to decide whether the presented item was the name of an animal. Because it was important to establish that the nonwords used in this experiment showed an inhibitory effect in lexical decision, the set of nonwords used in Forster and Shen (1996) was used in this experiment. These nonwords exhibited a robust inhibitory effect of $N$ in that lexical decision experiment. Because the task was the same as that used in Forster and Shen, it was decided to include the same set of animals and non-animals as used in that earlier study.

\section{Method}

Participants. Twenty-one undergraduate students enrolled in an introductory psychology course at the University of Arizona participated in the experiment as part of their course requirement.

Materials and Design. A total of 117 items of five to six letters in length were used. These consisted of 39 exemplars (i.e., animal names), and 78 nonexemplars. The nonexemplars were taken from Forster and Shen (1996, Experiment 2). These consisted of 39 words (e.g., vogue) and 39 orthographically legal nonwords that differed from a nonexemplar word by one letter (e.g., walley). All items were subdivided into three equal groups according to the number of neighbors. Items in the first group had only one neighbor, items in the second group had two neighbors, and items in the third group had three or more neighbors (mean number $=3.7$ ). Because of the difficulty of finding animal names with the desired number of neighbors, it was not possible to equate the exemplars in each condition for typicality or frequency. The complete set of items is listed in the Appendix.

Procedure. The stimuli were presented on a color monitor using the Windows-based DMDX software package developed by J. C. Forster at the University of Arizona (for details, see the website at psy1.psych.arizona.edu/ kforster/dmdx/index.htm). This system synchronizes the display program with the video raster, enabling accurate measurements of reaction time (RT). The display consisted of black characters on a white background. The items were presented in a different pseudorandom order for each participant. The entire list of items was divided into blocks, with each block containing an equal number of items from each condition. The order of items within each block was then randomized, as were the blocks. To begin each trial, the participant pressed a foot pedal. Immediately after, a fixation cross appeared in the center of the screen for $500 \mathrm{msec}$ followed by the target item for $500 \mathrm{msec}$ displayed in uppercase letters (a standard method for indicating a target in our laboratory). Participants responded by pressing the "yes" key with the right hand or the "no" key with the left hand. After the response, a feedback message was presented specifying the speed and accuracy of the response. The instruction given to the participants was to decide whether the letter string presented was an animal name or not. They were specifically warned about the presence of nonwords and told to respond "no" to such items. Both accuracy and speed of performance were stressed. There were 15 practice items. Any participants making more than $20 \%$ errors were replaced.

Data treatment. In this experiment, and all subsequent experiments, the data from trials on which an error occurred were discarded. The mean RTs for correct responses and the average error rates in each condition were calculated separately across participants and items, and each data set was subjected to a separate analysis of variance (ANOVA) ( $F_{1}$ for participants, $F_{2}$ for items). An alpha level of .05 was adopted for all analyses. Prior to this analysis, RTs for each individual were trimmed by establishing cutoff points $2 S D$ units above and below the mean for that individual. Any RTs exceeding the cutoff value were set equal to the cutoff, rather than being excluded from the analysis. This procedure was thought to be more conservative, since the focus of interest in these experiments was an inhibitory effect, and simply excluding long RTs altogether might have minimized this effect. 1

\section{Results}

Data trimming resulted in the modification of less than $4.9 \%$ of the data points. One participant had to be replaced because the error criterion of $20 \%$ was exceeded. The mean RTs are shown as a function of $N$ in Table 1.

RT analyses of the data for the nonwords revealed no main effect of $N$ for nonwords $\left[F_{1}(2,40)<1, F_{2}(2,24)<1\right]$. Not only were the differences unreliable, but they were not in the expected direction, since an increase in $N$ from 1 to 2 produces a small increase in speed, and there is no difference between items with an $N$ of 3 and items with an $N$ of 1 . Similarly, there was no main effect of $N$ for the nonexemplar words $\left[F_{1}(2,40)<1, F_{2}(2,24)<1\right]$, with the means in the three conditions differing only by $3 \mathrm{msec}$. The error data for the nonexemplars showed a similar pattern. There was no significant effect of $N$ for nonwords (both $F \mathrm{~s}<1$ ) or for nonexemplar words $\left[F_{1}(2,40)=2.88, M S_{\mathrm{e}}=41.5\right.$, $\left.F_{2}(2,24)<1\right]$. Because of the difficulty of controlling for typicality and frequency, the data from the exemplars are not typically reported. However, it is worth noting that there was a significant negative correlation between esti-

Table 1

Experiment 1: Mean Semantic Categorization Times (RT, in Milliseconds) and Percentage Error Rates (PE) for Nonwords and Nonexemplar Words as a Function of Number of Neighbors $(N)$

\begin{tabular}{cccl}
\hline Item & RT & PE & $N$ \\
\hline Nonwords & & & \\
ramin & 612 & 3.3 & 1 \\
durate & 592 & 3.3 & 2 \\
poler & 611 & 4.4 & $3+$ \\
Words & & & \\
piston & 585 & 5.5 & 1 \\
legion & 588 & 3.3 & 2 \\
impact & 585 & 8.1 & $3+$ \\
\hline
\end{tabular}


mates of typicality (Battig \& Montague, 1969) and categorization time $[r(38)=-0.35, p<.05]$.

\section{Discussion}

The results indicate that there was no trace of any interference effect of $N$ for nonwords, even though the same items had shown a robust inhibitory effect of $68 \mathrm{msec}$ in an earlier lexical decision experiment (Forster \& Shen, 1996). This result is problematic for form-first verification models that treat the $N$ effect as intrinsic to the process of word recognition. In these models, any decision about a nonword would have to be delayed until all lexical candidates had been eliminated.

A subsidiary finding was that $N$ had no effect for nonexemplar words. This result replicates the finding of Forster and Shen (1996), which is not surprising because exactly the same items were used. However, both Carreiras et al. (1997) and Sears et al. (1999) have pointed out that the manipulation of $N$ in the Forster and Shen paper was not particularly strong. Both groups reported significant effects of $N$ for words in a semantic categorization task when the high- $N$ group had a mean of approximately 7 neighbors (compared with a value of 3.7 in the present experiment). This possibility cannot be overlooked, although it must be pointed out that a similar manipulation of $N$ was sufficient to produce clear effects in a lexical decision task (Forster \& Shen, 1996, Experiment 2). Nevertheless, it might be argued that the tasks involve different components and that a stronger manipulation of $N$ might have produced a different result. However, it is important to note that the effects reported by Carreiras et al. and Sears et al. are not the same. Carrieras et al. reported an inhibitory effect of higher frequency neighbors in the case of words with many neighbors, but no overall effect of $N$, whereas Sears et al. reported a facilitatory effect of $N$, but no overall effect of higher frequency neighbors. So it is not clear what to expect if a stronger manipulation of $N$ had been used here. Also, it should be pointed out that it would be difficult to argue that there really is an effect for words while at the same time accepting that there is no effect for nonwords.

For present purposes, the more important result is that for nonwords there appears to be no effect at all, which is inconsistent with any model that assumes a verification process. However, this is exactly the result that a semantic feature monitoring model such as that proposed by Carreiras et al. (1997) would predict. In such a model, a "no" decision is made when a deadline expires without activation in the critical semantic feature reaching criterion. Activation in the units for neighbors would be irrelevant because the decision procedure is tuned to activity at the semantic level and ignores activity at the word level.

The critical feature of this model is that activation is cascaded. Within a parallel activation model, cascaded activation of semantic properties is easily implemented, but this is more difficult in a form-first model such as the entry-opening model. In such a table lookup model, semantic properties cannot be retrieved until the correct lex- ical entry is found. Nor is it possible to explain the results by introducing a cascading assumption. For example, if the model were modified so that the semantic properties of each candidate could be extracted and verified before the correct candidate had been located, one would still expect an $N$ effect for nonwords. Each such verification would take time, and therefore the more candidates that had to be verified, the longer the "no" decision should take. What this demonstrates is that the evaluation process would have to be a parallel process.

At an intuitive level, the results suggest that the number of close-match candidates might be relevant only for making a decision about form, as would be the case in lexical decision. In such a task, the only relevant property of the stimulus is its spelling, and hence it is not surprising that orthographic form needs to be checked before a decision can be made. However, when a decision about meaning is being made, the accuracy of the spelling may be ignored altogether. That is, when meaning is the focus of the task, orthography becomes less important, especially when the correct answer is "no," because it is irrelevant whether the stimulus turns out to be a word or a nonword-in either case, a "no" decision is appropriate. McRae and Boisvert (1998) reported a phenomenon compatible with this analysis. In a lexical decision task, error rates on words with unusual spelling patterns (e.g., tomahawk, bazooka) were quite high, although these words were correctly classified in a semantic categorization task, where all stimuli were words, and hence the accuracy of spelling was not at issue. This view of the decision process is well captured by a model such as MROM, which allows for decisions to be controlled by activation patterns at different levels.

However, there are other possible interpretations. For example, the absence of the nonword $N$ effect in semantic categorization is also consistent with the assumption that the effect in lexical decision is really due to a decision bias. This bias could be based on the fact that high- $N$ items seem more "wordlike" because they are more likely to contain common orthographic units (e.g., bodies). ${ }^{2}$ This bias produces opposite effects for words and nonwords in lexical decision. But such a bias would be inoperative in a semantic categorization task, because animal names (presumably) are not more wordlike than other words. On this interpretation, $N$ has no direct effect in lexical decision, but is merely correlated with the true causal variable, namely "wordlikeness." Thus, the properties of individual neighbors play no role at all in word recognition.

This argument leads to an obvious question. What would happen if one of the neighbors was in fact a member of the category? If the properties of neighbors are completely ignored, then nonwords based on animal names (e.g., turple, whele, eigle) should be rejected just as rapidly as any other nonword. However, for a cascaded feature monitoring model, there should be substantial interference, because nonwords such as turple will activate the appropriate semantic feature (because turple activates the word unit for turtle). The next experiment was designed to test whether this is the case. 


\section{EXPERIMENT 2}

To test whether there is an interference effect for nonwords that have an animal name as a neighbor, the ideal design would be to vary $N$ over a range from 1 to 3 , as was done in Experiment 1. However, this involves finding a reasonable number of nonwords that have several animal names as neighbors, which is extremely difficult. Instead we were forced to consider items with a single animal name as a neighbor. To test for an $N$ effect, these items could be compared with nonwords that had no neighbors at all (e.g., slicht), but here there is a danger of confounding $N$ with orthographic factors, because items with no neighbors are more likely to involve unusual letter sequences. Instead we compared these items with nonwords that were also one-letter-different from a word, but one that was not an animal name (e.g., cishop). Given the results of Experiment 1 , such items should not exhibit any interference effect, and hence could serve as an adequate baseline. The fact that both types of nonwords are derived from real words offers some assurance that the two sets are comparable, but it by no means guarantees equivalence.

\section{Method}

Participants. Thirty-two undergraduate students enrolled in an introductory psychology course at the University of Arizona participated in the experiment as part of their course requirement.

Materials and Design. A set of 160 items of 5 to 9 letters in length was used, consisting of 80 exemplars (animal names) and 80 nonexemplars. Half of the nonexemplars were words, and half were nonwords. The 40 nonwords were constructed either by changing one letter of an animal name (e.g., turple, whele, mooster) or by choosing a word of similar frequency that was not the name of an animal (e.g., kinse, accest, tabric). The overall mean frequency of the animal base words was 9.2 per million; for the non-animal base words, it was 10.7 per million. Owing to a transcription error, one of the nonwords in this condition was two letters different from a word and was omitted from the analysis along with its corresponding nonword based on an animal name, leaving 19 items in each condition. The nonexemplar words consisted of 20 high-frequency words (between 150 and 300 counts per million in Kučera \& Francis, 1967) and 20 low-frequency words (no more than 10 counts per million). The complete set of items is listed in the Appendix.

Procedure. The procedure and task were the same as in Experiment 1 .

\section{Results}

The mean RTs and errors for nonwords and the nonexemplar words are shown in Table 2. Seven participants were replaced for exceeding the $20 \%$ error criterion, and $3.5 \%$ of the data points were replaced by the trimming procedure. Analysis of the RTs for the nonwords showed a significant difference $\left[F_{1}(1,31)=110.9, M S_{\mathrm{e}}=1099.2\right.$, $\left.F_{2}(1,18)=30.7, M S_{\mathrm{e}}=3554.7\right]$. RTs to reject items like turple were $87 \mathrm{msec}$ longer than for items like cishop. A similar pattern was observed for the error data. The error rate of $22.7 \%$ for the turple items was significantly larger than the $5.9 \%$ error rate for the cishop items $\left[F_{1}(1,31)=63.5, M S_{\mathrm{e}}=70.9, F_{2}(1,18)=17.6, M S_{\mathrm{e}}=\right.$ 152.4]. Analysis of the RT data for the nonexemplar words showed that high-frequency words were responded to
$29 \mathrm{msec}$ faster than were low-frequency words. This frequency effect was significant $\left[F_{1}(1,31)=17.45, M S_{\mathrm{e}}=\right.$ 793.0, $\left.F_{2}(1,19)=7.9, M S_{\mathrm{e}}=1,117.0\right]$. Overall there was no difference in errors between high- and low-frequency words (both $F_{\mathrm{s}}<1$ ).

\section{Discussion}

Nonwords with an animal name as a neighbor, such as turple, took much longer to categorize and produced a far higher error rate than nonwords with a nonexemplar neighbor such as cishop. This result is not particularly surprising, but nevertheless demonstrates two things. First, interference effects from a neighbor can be detected in a semantic categorization task, so it is not simply the case that the task itself obscures any effect. Second, this result makes it clear that the semantic properties of individual neighbors are taken into account before a decision is made, but in such a way that semantically irrelevant candidates do not slow the decision process. The implication of this result is that the decision process must employ an initial semantic test, so that only the candidates that have the correct semantic properties produce any delay in the decision (whether by extension of a deadline or by verification of candidates). The fact that the semantic properties of individual neighbors are being taken into account indicates that neighbors do more than simply increase global activation levels; they play an important role in word recognition.

At first, it appears that this result conflicts with the findings of Bourassa and Besner (1998), who found that in lexical decision, nonwords based on a word (e.g., tuble) were not capable of priming a target word semantically related to the base word (e.g., CHAIR) when the prime was visible, although a small effect was observed when the prime was masked. The fact that no priming occurred for visible primes suggests that a nonword such as tuble does not activate the semantic properties of its base word. However, these two situations are not strictly comparable, because the tasks differ (lexical decision vs. semantic categorization), and the type of effect differs (facilitation vs. interference). Further, the fact that no semantic priming occurred does not necessarily imply that there was no semantic activation. Nevertheless, it is interesting to consider how these effects can be reconciled. It could be that semantic priming is produced by a decision strategy that

Table 2

Experiment 2: Mean Semantic Categorization Times (RT, in Milliseconds) and Percentage Error Rates (PE) for Nonwords With and Without an Animal Neighbor and for Nonexemplar Words Varying in Frequency

\begin{tabular}{lllr}
\hline \multicolumn{1}{c}{ Condition } & Example & RT & PE \\
\hline Nonwords & & & \\
$\quad$ Exemplar neighbor & turple & 750 & 22.7 \\
$\quad$ Nonexemplar neighbor & cishop & 663 & 5.9 \\
Words & & & \\
$\quad$ High-frequency nonexemplar & train & 612 & 3.6 \\
Low-frequency nonexemplar & feast & 641 & 3.6 \\
\hline
\end{tabular}


is disrupted when the prime is consciously perceived to be a nonword. Another possibility is that by the time information about the prime reaches consciousness, the semantic activation produced by tuble is inhibited, unless the task places special emphasis on semantic properties. Thus, there is insufficient activation to produce priming in lexical decision, but sufficient activation to produce interference in semantic categorization.

The result of this experiment, like the absence of an $N$ effect in Experiment 1, is again incompatible with formfirst models, which assume that semantic properties are unavailable prior to resolution at the level of form. On the other hand, the result is easily explained by a cascaded feature monitoring model. Nonwords such as turple must activate the features appropriate for a "yes" decision, but not to criterion level. When such activation is detected, the deadline for a "no" decision is extended to allow for the possibility that the stimulus is a very low frequency animal name. This extension of the deadline leads to a slower response.

Before considering the adequacy of this explanation, we must address a methodological issue. It could be that, in this experiment, there was an accidental confound with the similarity of the nonword to its base word. That is, the reason that turple takes longer than cishop may be just that turple is more similar to turtle than cishop is to bishop. To assess whether this is the case, we could attempt to measure the similarity of each nonword to its base word. However, this would require a detailed theory of orthographic similarity, which we do not have. Obtaining ratings from judges is not an option either, because this presupposes that the judges are sensitive to the perceived similarity of a letter string to a word during the first few hundred milliseconds of exposure.

Certainly, at a phenomenal level, the base words of the animal group seem far more obvious (see the item listings in the Appendix). For example, it is hard to imagine that the similarity of kandaroo to kangaroo would go unnoticed, possibly because this is a relatively long word, or because it contains relatively unusual letter sequences. On the other hand, the control item for this nonword, ecstatin, was based on a word of similar length and frequency $(e c$ static), which also contains relatively unusual letter sequences. Nevertheless, it could be argued that the speed with which the base word is recovered might vary, perhaps because ecstatic is an adjective whereas kangaroo is a noun. This was true for 6 of the 13 items and resulted from the fact that the nonexemplar base words were chosen strictly at random (in an attempt to avoid a selection bias). However, it seems highly unlikely that grammatical category could play any role, since it is generally assumed that any interference generated by a neighbor is controlled by form and form alone. Other examples of "obvious" animal neighbors are elephint, naccoon, yebra, and torboise. In contrast, it is sometimes extremely difficult to determine the base words for some of the control nonwords (e.g., noole, inpale, mahor, traipte).
Two points need to be made about these observations. First, the reason that the animal base words seem more obvious may be because we know that we are looking for animal names. Given similar semantic cues, the base words for the control nonwords might be just as obvious (e.g., the base word for mahor suddenly becomes clear when the category military rank is provided). So this may not be a fault in the design. In fact, this phenomenon is precisely what the experiment was designed to show-that the base word is more obvious when we know its likely semantic (or perhaps even syntactic) category. The second point is that the time taken for conscious recognition of the base word may be a poor indicator of similarity. For example, it is very difficult to identify the neighbors of some words (e.g., what is the neighbor of backward?), yet these words are primed by their neighbors perfectly well in a masked priming paradigm where the interval between prime and target onset is very brief (Forster \& Veres, 1998). In addition, measurements of the time taken to identify the base word (over $3,000 \mathrm{msec}$ ) indicate that conscious recognition occurs far too late to have any effect on lexical decision times (Chambers, 1976).

This impasse can be circumvented simply by comparing responses to the turple and cishop items in a lexical decision task, where the semantic properties of the neighbors are not relevant. If it is the case that similarity was not controlled in Experiment 2, or that the base words differed in accessibility, then we ought to observe the same difference in lexical decision times (i.e., turple items should be harder to reject than cishop items). The next experiment investigates whether this is the case.

\section{EXPERIMENT 3}

If the effects observed in Experiment 2 were due to inadequate control of the similarity of the nonword to its base word, then we should expect to find similar interference effects in a lexical decision task. That is, the nonwords with an animal as a neighbor (turple) should take substantially longer to classify than the nonwords with a non-animal as a neighbor (cishop). In addition, a baseline condition was included consisting of nonwords without any neighbors to check that the experiment was sufficiently sensitive to pick up the interference caused by a neighbor.

\section{Method}

Participants. Twenty-five undergraduate students enrolled in an introductory psychology course at the University of Arizona participated in the experiment as part of their course requirement.

Materials and Design. The critical nonwords were taken from Experiment 2. These consisted of the same 20 turple nonwords and 20 cishop nonwords, together with a further set of 20 nonwords that had no neighbors (e.g., glimon, tamal, esporn). These were included as a baseline condition. To disguise the large number of nonwords that closely resembled animal names, an additional 40 nonwords (e.g., byme, tetch, crube) were included as fillers, making a total of 100 nonwords. Also included were 100 arbitrarily chosen words matched with the nonwords for length. 
Table 3

Experiment 3: Mean Lexical Decision Times (RT, in Milliseconds) and Percent Error Rates (PE) for Nonwords with an Animal Name or a Non-animal Name as a Neighbor

\begin{tabular}{lllr}
\hline \multicolumn{1}{c}{ Condition } & Example & RT & PE \\
\hline Animal neighbor & turple & 748 & 17.0 \\
Non-animal neighbor & cishop & 735 & 12.8 \\
No neighbor & rodis & 687 & 4.2 \\
\hline
\end{tabular}

Procedure. The procedure was the same as in Experiment 1, except that the task was to decide whether the item was a word.

\section{Results and Discussion}

The mean lexical decision latencies and error rates for the nonwords are shown in Table 3. A one-way ANOVA performed on the mean RTs for the three nonword conditions revealed a significant effect $\left[F_{1}(2,48)=8.58, M S_{\mathrm{e}}=\right.$ $\left.3,021.1, F_{2}(2,57)=6.74, M S_{\mathrm{e}}=5,505.6\right]$, demonstrating that the presence of a neighbor slowed decision times. This effect was on the order of $61 \mathrm{msec}$ if the neighbor was an animal name, and $48 \mathrm{msec}$ if it was not. This difference of $13 \mathrm{msec}$ in the two $N$ effects might be taken as an indication that the nonwords used in Experiment 2 were not adequately matched for similarity to the base word. However, this difference was not significant (both $\left.F_{1}, F_{2}<1\right)$. There was a trend in the error rates in the subject analysis $\left[F_{1}(1,24)=4.10, M S_{\mathrm{e}}=53.8, p=.054\right]$ but not in the item analysis $\left[F_{2}(1,38)=1.15, M S_{\mathrm{e}}=152.0\right.$, $p>.05]$.

The critical point is not so much whether the two sets of nonwords were perfectly equivalent, but rather whether the semantic categorization task amplified whatever difference there was in a lexical decision task. To establish this, a $2 \times 2$ ANOVA was performed comparing the mean RTs for the turple and cishop items in the semantic categorization task (Experiment 2) with the means for the same items in the present lexical decision experiment. This item analysis produced a significant interaction between task and type of neighbor $\left[F_{2}(1,36)=7.53, M S_{\mathrm{e}}=\right.$ $4,051.0]$. Hence it can be concluded that the semantic categorization task produced a larger effect.

An additional check is to use the lexical decision data to select a set of items matched on overall lexical decision time and then determine how they performed in the semantic categorization task. If we discard the three slowest items in the turple condition (elephint, kandaroo, and speep), the mean lexical decision times for the two groups of nonwords are virtually identical (745 vs. $743 \mathrm{msec})$. However, the same items showed an 86-msec effect in the semantic categorization task ( 739 vs. $653 \mathrm{msec}$ ), this effect being significant in the item analysis $[t(34)=6.34]$.

\section{EXPERIMENT 4}

It seems clear enough that the strong interference produced by an animal neighbor in Experiment 2 was not due to the fact that the nonwords with an animal neighbor (turple) bore a closer resemblance to their base word than the nonwords without an animal neighbor (cishop). Nor could it be the case that the turple items were more "wordlike" than the cishop items, because this would also have produced a lexical decision difference. Thus it seems clear that the results favor a feature monitoring strategy, in which the deadline for a "no" decision is extended when substantial activation in the animal feature is detected. One might try to rescue the form-first verification models by suggesting that each candidate is subjected to a very limited semantic test, sufficient to establish whether it is likely to be an animal name, but not much else. The effect would be that only candidates that are animal names need to be checked. However, this proposal leads to a subtle problem. How is it established that a candidate is or is not an animal name, without applying a semantic test to each candidate? Such a test must take time, and therefore there should still be an $N$ effect, although perhaps smaller in size than in lexical decision. We return to this issue in the General Discussion.

Although the results so far clearly favor a cascaded model, several problems remain to be resolved. First, as indicated earlier, "no" decisions are generated when a deadline expires and there is insufficient activation in the appropriate semantic features. To explain the effect for turple nonwords (Experiment 2), it must be assumed that the deadline is extended when weak activation of the animal feature is detected. However, the use of a deadline mechanism for "no" responses creates at least two problems. The first problem is that there should be no difference in RTs between nonexemplar words and nonwords without an animal neighbor. In either case, the decision is made when the deadline expires. However, inspection of the data in Tables 1 and 2 suggests that this is not the case: The nonwords take on average $26 \mathrm{msec}$ longer. The second, and more severe problem, is that the assumption of a deadline makes it difficult to explain the existence of a frequency effect for nonexemplar words. Such an effect was observed in Experiment 2, and similar effects have been reported elsewhere for semantic categorization (Forster \& Shen, 1996; Monsell, Doyle, \& Haggard, 1989). If decisions are based purely on a deadline, then it should be set sufficiently long to allow low-frequency animal names to activate the appropriate semantic features to some criterion level. Hence both high- and low-frequency nonexemplars should yield equivalent decision times. Clearly, this deficiency must be addressed.

Carreiras et al. (1997) recognized that if decisions in a semantic categorization task were made by a cascaded feature monitoring system, then "no" decisions must be generated by the expiration of a deadline, and hence no variation in decision times as a function of neighborhood characteristics would be expected for nonexemplars. Yet they observed an interaction between $N$ and the presence of higher frequency neighbors for nonexemplar words, such that there was an inhibitory effect of $N$ for words with a higher frequency neighbor, but not for words without a higher frequency neighbor. Carreiras et al. attempted to explain this by arguing that the nonexemplar words must 
Table 4

Experiment 4: Mean Semantic Categorization Times (RT, in Milliseconds) and Percentage Error Rates (PE) for High- $N$ and Low- $N$ Nonwords With One Animal Neighbor, and for Nonexemplar Words

\begin{tabular}{lccr}
\hline Nonexemplars & Example & RT & PE \\
\hline High- $N$ nonwords & goan & 631 & 14.2 \\
Low- $N$ nonwords & cadel & 644 & 12.4 \\
Zero- $N$ nonwords & thowl & 607 & 1.2 \\
Words & sugar & 600 & 8.7 \\
\hline
\end{tabular}

have activated the animal features to varying degrees (despite being nonexemplars), citing the fact that categorization RTs and error rates were weakly correlated with ratings of "animalness."

The implication of this argument is that if a relatively high proportion of items in the experiment activate the relevant semantic features, a "no" decision is always delayed until resolution at the level of form is completed. This could be achieved by adopting a dual monitoring system, in which global activation at the word level is also monitored. For nonexemplar words, a "no" decision could be generated before the deadline expires if the global activation level is very high, but there is little or no semantic activation in the animal feature, on the grounds that this feature should have reached criterion by this time if the word was an animal name. But if the global activation level is low, no decision can be made until the deadline expires. This will generate a frequency effect for nonexemplar words because high-frequency words will drive the global activation index up to the required level more rapidly than low-frequency words will.

This approach raises an interesting possibility for the present line of research. With a dual monitoring scheme, a nonword $N$ effect may be observed in a semantic categorization task if at least one of the neighbors is an exemplar. The reasoning is as follows. The presence of an animal neighbor means that the nonword will activate the animal feature, but not sufficiently to trigger a "yes" decision. Under these conditions, a decision is delayed until there is some resolution at the level of form, using the global activation index as a guide. If the global activation level is very low, then it is possible that the stimulus is a nonword, and therefore a deadline of normal length is used. However, if global activation is at an intermediate level, an extension to the deadline may be required in case the input is a very low frequency animal name that has not yet reached the global activation level typical of words. High$N$ nonwords are more likely than low- $N$ nonwords to produce such an intermediate activation level, and therefore an inhibitory effect of $N$ should be observed. Note that this hypothesis is different from the hypothesis tested in Experiment 2. In that experiment, we tested whether a nonword with an animal neighbor would take longer to reject than a nonword with a non-animal neighbor. In the present experiment, both the high- $N$ and low- $N$ nonwords have an animal neighbor, but they vary in the number of non- animal neighbors. If activation of the animal feature delays a "no" decision until resolution at the level of form occurs, then the number of non-animal neighbors should be relevant.

\section{Method}

Participants. Twenty-two undergraduate students enrolled in an introductory psychology course at the University of Arizona participated in the experiment as part of their course requirement.

Materials and Design. A total of 45 nonwords served as critical items. These were divided into three sets: (1) 15 high- $N$ nonwords with one animal neighbor (e.g., swark, duve, fown, bealer, mean $N=$ 6.9), (2) 15 low- $N$ nonwords with one animal neighbor (e.g., swen, eaple, toger, oyl, tartle, mean $N=2.2$ ), and (3) 15 nonwords with no neighbors at all (e.g., thowl, notrix, atova, daran, dagen). In addition, there were 45 nonexemplar words matched with the nonwords for $N$ and length. Ninety animal names were used as exemplars, none of which were neighbors of the nonwords.

Procedure. The task was an animal categorization task, as in Experiment 1 . All aspects of the procedure were the same as in Experiment 1.

\section{Results and Discussion}

The mean RTs and error rates are shown in Table 4. The first result of note is that high- $N$ nonwords with an animal neighbor did not take longer to reject than low- $N$ nonwords with an animal neighbor-in fact they were $13 \mathrm{msec}$ faster, but this difference was nowhere near significant $\left[F_{1}(1,21)=1.86, M S_{\mathrm{e}}=939.8, F_{2}<1\right]$. Second, zero- $N$ nonwords clearly were much easier to reject than low- $N$ nonwords, both in RTs $\left[F_{1}(1,21)=33.36, M S_{\mathrm{e}}=451.4\right.$, $\left.F_{2}(1,14)=15.15, M S_{\mathrm{e}}=717.4\right]$ and error rates $\left[F_{1}(1,21)=\right.$ $\left.32.00, M S_{\mathrm{e}}=43.2, F_{2}(1,14)=16.83, M S_{\mathrm{e}}=56.0\right]$, and they were also easier to reject than high- $N$ nonwords, both in RTs $\left[F_{1}(1,21)=7.62, M S_{\mathrm{e}}=860.3, F_{2}(1,14)=5.95\right.$, $\left.M S_{\mathrm{e}}=955.1\right]$ and error rates $\left[F_{1}(1,21)=33.31, M S_{\mathrm{e}}=\right.$ $\left.56.0, F_{2}(1,14)=14.17, M S_{\mathrm{e}}=89.9\right]$, indicating that the experiment had sufficient power to detect the effect of the neighbor.

From the fact that nonwords with an animal neighbor took longer to reject than both the zero- $N$ nonwords and the matched nonexemplar words, we can infer that the existence of the animal neighbor was detected. However, it seems clear that the number of non-animal neighbors plays no role. This result is not consistent with the view that the presence of an animal neighbor causes the decision to be delayed until resolution occurs at the level of form, because the number of non-animal neighbors should then influence resolution time.

At a purely informal level, what appears to be happening is that the presence of a single animal neighbor does indeed delay any decision until the spelling of that word can be compared with the input, but only that one word needs to be checked. The other neighbors are ignored. What needs to be explained is how the lexical processing system knows which candidate to check. In the kind of dual monitoring system that we have been considering, all that is known is that the relevant semantic feature is active and that the global activation index is either high or low, de- 
pending on $N$. There is no way to explain why extension of the deadline should be the same regardless of the number of neighbors.

\section{GENERAL DISCUSSION}

The general implications of these findings are clear. The absence of any $N$ effect for nonwords in semantic categorization (Experiment 1) is inconsistent with the assumption that resolution at the level of form must be completed before any semantic information can be retrieved. Either one assumes that some type of semantic information is available before resolution at the level of form, or one concludes that the nonword $N$ effect in lexical decision has nothing to do with this process and simply reflects a decision bias.

Although decision bias explanations are notoriously difficult to eliminate, it appears that the neighbor effect observed for the turple nonwords in Experiment 2 goes some way toward achieving this goal. The decision bias argument assumes that in lexical decision, the bias is based on factors such as perceived familiarity, orthographic redundancy, wordlikeness, and so on. It is not based on the presence of particular neighbors. The relevance of $N$ comes about purely because $N$ is correlated with these factors. However, the turple effect is clearly produced by the presence of a neighbor with a particular semantic property and has nothing to do with extraneous factors such as wordlikeness, as evidenced by the fact that lexical decisions for these nonwords were comparable to those of nonwords with a single non-animal neighbor (Experiment 3). Hence we conclude that the turple effect demonstrates that the presence of actual neighbors is a factor in word recognition.

Some aspects of this effect are readily explained by a cascaded feature monitoring model because the assumption of cascaded activation makes it possible for semantic decisions to be made independently of any neighborhood effects. This explains the absence of an $N$ effect for nonwords in Experiment 1. If we assume that the deadline for making a "no" decision is extended when appropriate semantic activation is detected, it is also possible to explain the longer RTs for the turple nonwords in Experiment 2. However, what is difficult to explain is the existence of a frequency effect for nonexemplar words, because the deadline would be the same for both types of words. A similar argument applies to the neighborhood effects observed by Carreiras et al. (1997). A dual monitoring scheme, in which weak activation of the critical semantic feature triggers an additional monitoring of global activation levels, could explain these effects. However, the results of Experiment 4 provide no support for such a scheme. This model predicts that for nonwords that have an animal neighbor, the deadline for a "no" decision should be extended when there is also a large number of nonanimal neighbors, but the results indicate clearly that this is not the case.
Given these difficulties, it is perhaps worth considering whether there is some other method of achieving a "semantics-first" strategy. One possibility is to abandon the deadline procedure for making a "no" decision for words and to propose that activation at the semantic feature level can support both "yes" and "no" decisions. This would require the existence of bipolar features, where positive levels of activation indicated the presence of the feature, and negative values indicated the absence of the feature. ${ }^{3}$ It could then be argued that exemplars would generate positive activation, and nonexemplars would generate negative activation, but in each case, the rate at which activation is produced is a function of frequency.

Essentially the same proposal has been put forward by Plaut (1997) in his semantic "stress" model, which allows a distributed network model without word units to make lexical decisions. In a similar fashion to the bipolar model discussed above, words that possess a semantic attribute drive the activation level of the appropriate semantic unit from a resting level of 0.5 up toward a value of 1 , and words that do not possess that attribute drive activation down to a level of 0 . The stress of a unit is defined as 0 when it is at rest, and approaching 1 as the activation level approaches either 1 or 0 . Plaut assumes that although nonwords activate the semantic units appropriate to their neighbors, these features may conflict with each other (e.g., manmade and animate), and hence nonwords will not drive semantic units as efficiently as words. By summing the stress values of all semantic units, Plaut shows that reasonably accurate lexical decisions can be made.

This account is very similar in spirit to the global activation account employed in MROM (Grainger \& Jacobs, 1996) and DRC (Coltheart et al., 2001), and hence presumably predicts that high- $N$ nonwords should take longer to classify than low- $N$ nonwords in lexical decision. In a semantic task, the appropriate stress value would be summed over just the category-relevant units rather than all semantic units, and because changes in activation occur more rapidly for high-frequency words than for lowfrequency words, both "yes" and "no" decisions for words will show a frequency effect. Because nonwords will presumably generate intermediate activation levels (i.e., not enough positive activation to generate a "yes" decision and not enough negative activation to generate a "no" decision), something equivalent to a deadline mechanism would still be required, and the neighbor effect obtained for turple items in Experiment 2 could be handled by extensions to the deadline when weak positive activation is detected. Thus, all of the effects reported here could be accommodated within a model of this type.

An obvious weakness of this approach is the assumption that a given word must be coded on every semantic attribute that it does not possess. That is, if the animal category is represented by a bipolar feature, then all animal names will have to be connected to this feature with positive weights, and all other words will have to be connected to this feature with negative weights. Exactly the same is 
true for the stress model, which must be trained to either raise or lower the activation level in every semantic unit. This might be plausible for very general properties, such as abstract-concrete, or animate-inanimate, but is less plausible for more specific categories, such as "article of clothing" or "type of vehicle." That is, words such as desk or evening would have to be coded as not-clothing, notvehicle, not-edible, not-liquid, not-number, and so on. Because the number of features required to code the meanings of all words might be very large indeed, this solution involves a huge number of additional connections, the sole purpose of which is to account for the frequency effect for nonexemplars. Furthermore, these connections are completely redundant because they provide the same information as the absence of any positive connection. Thus it might be more sensible to limit the use of bipolar features to very general semantic properties, such as animacy. In this case, one would predict that frequency effects for nonexemplars should only be found for very general properties. No such effect would be expected for specific categories such as "article of clothing." This hypothesis has not yet been tested.

So far, we have given little attention to the question of whether it is possible to modify a form-first verification model such as the entry-opening model so that it can accommodate these results. To preserve the form-first postulate, it is necessary to find some way to achieve selective form checking of candidates. One possibility is that the properties that govern whether a candidate is submitted to a form check do not involve activation of semantic features as such. Instead, this decision could be based on information provided by a network of associative links between lexical entries and conceptual structures, or semantic fields, in a manner similar to a thesaurus. These links may serve as indicators of possible category membership, but do not provide detailed semantic information. Thus, the entries for all animal names could be linked with a conceptual structure that corresponds to an animal semantic field. When a nonword with an animal as a neighbor is presented (e.g., pitten), the initial stage of processing based on form (a frequency-ordered search in the case of the entry-opening model) locates the entries for the word kitten, as well as the entries for mitten and bitten, which are all flagged as candidates. As each candidate is flagged, the associative links to semantic fields become active. These links reveal that kitten is a possible animal candidate (because it is linked to the animal field), but that mitten and bitten are not. A form check is then carried out to determine whether the entry for kitten actually matches the input and because it does not, a "no" decision is made without requiring further checks on mitten and bitten. If the stimulus had actually been kitten, then the form check would have been successful, but a further check of the semantic properties of kitten would have been required before a "yes" decision could be made. The reason for this is that the associative links are purely heuristic devices, and are error-prone.
This model predicts that, for nonwords, a decision will be delayed until every candidate that is linked with the animal semantic field has been eliminated. Thus a "no" decision to a nonword such as turple will be delayed until the candidate entry for turtle has been eliminated by a form check, which explains the neighbor effect observed in Experiment 2. But for nonwords such as insate, no neighbor effect is expected, because none of the candidates (insane, inmate, and innate) is linked to the animal field. Further, in Experiment 4, this model explains why there is no effect of variation in $N$ when one of the neighbors is an animal. The link information signals which candidate needs to be checked, as in the pitten example, and there is no need to check the remaining neighbors because they lack the relevant links.

However, as indicated earlier, there is still the problem that the links for each candidate have to be checked, and this should generate an $N$ effect because it must take time to establish whether there is or is not a link to the animal field. Attempting to check all links in parallel is not an option because it is necessary to find out which candidate is the one with the critical link in order to check it. The solution to this problem is to propose that the links can be processed fast enough to keep pace with the search process, so that at any one time, only one link needs to be considered, and provided that it can be dealt with before the next close-match candidate is discovered, there is no reason why several links should take longer to process than one.

The existence of a frequency effect for nonexemplar words (Experiment 2) is explained in the following way. Because these are words, the frequency-ordered search will find a matching entry, which is immediately checked for form and links. In the case of a nonexemplar, the appropriate link will be absent, and it is possible that a "no" decision could be generated at this point. Alternatively, the decision could be delayed until the semantic properties of the word have been activated. In either case, a major component of the decision time will be the time taken to locate the matching entry, which is controlled by frequency.

This network of associative links has obvious similarities to the model of semantic memory proposed by Collins and Quillian (1972). However, there are several important differences. The system proposed here operates before lexical identification, whereas the Collins and Quillian model presupposes that the word has already been identified. Further, no information is provided about the nature of the association. All that can be inferred from a link between the entry for turtle and the animal field is that these entities are related in some way. The reason for making this assumption is that the test for a possible relation with the category needs to be fast enough to keep pace with the search process; otherwise, bottlenecks are inevitable. This means that only very simple properties can be encoded.

Finally, it must be conceded that the links model and the feature monitoring model probably have more in com- 
mon than we have indicated so far. The links model maintains its form-first nature, but to explain the data, it is forced to postulate a special mechanism whereby it can detect likely semantic properties. The feature monitoring approach maintains its cascaded semantic-first approach, but to explain the data, it is forced to take into account information derived from the level of form. Hence, both models have to take into account information from both the level of form and the level of meaning simultaneously. Discriminating between these models clearly will require further research, although the evidence from Experiment 4 raises serious problems for the feature monitoring approach. Whether it will be possible to construct a pure semantics-only model for semantic categorization remains to be seen.

\section{REFERENCES}

ANDREwS, S. (1989). Frequency and neighborhoodeffects on lexical access: Activation or search? Journal of Experimental Psychology: Learning, Memory, \& Cognition, 15, 802-814.

ANDREWS, S. (1992). Frequency and neighborhoodeffects on lexical access: Lexical similarity or orthographic redundancy? Journal of Experimental Psychology: Learning, Memory, \& Cognition, 18, 234-254.

ANDREWS, S. (1997). The effect of orthographic similarity on lexical retrieval: Resolving neighborhood conflicts. Psychonomic Bulletin \& Review, 4, 439-461.

Battig, W. F., \& Montague, W. E. (1969). Category norms for verbal items in 56 categories: A replication and extension of the Connecticut category norms. Journal of Experimental Psychology Monographs, 80 (3, Pt. 2), 1-46.

Bourassa, D. C., \& Besner, D. (1998). When do nonwords activate semantics? Implications for models of visual word recognition. Memory \& Cognition, 26, 61-74.

Carreiras, M., Perea, M., \& Grainger, J. (1997). Effects of orthographic neighborhood in visual word recognition: Cross-task comparisons. Journal of Experimental Psychology: Learning, Memory, \& Cognition, 23, 857-871.

Chambers, S. M. (1976). The use of visual stimulus information in word recognition. Unpublished doctoral dissertation, Monash University, Melbourne.

Collins, A. M., \& Quillian, M. R. (1972). How to make a language user. In E. Tulving \& W. Donaldson (Eds.), Organization of memory (pp. 309-351). New York: Academic Press.

Coltheart, M., Davelaar, E., Jonasson, J., \& Besner, D. (1977). Access to the internal lexicon. In S. Dornic (Ed.), Attention and performance IV (pp. 535-555). New York: Academic Press.

Coltheart, M., Rastle, K., Perry, C., Langdon, R., \& Ziegler, J. (2001).DRC: A dual route cascaded model of visual word recognition and reading aloud. Psychological Review, 108, 204-256.

FoRSTER, K. I. (1999). The microgenesis of priming effects in lexical access. Brain \& Language, $\mathbf{6 8}, 5-15$.

Forster, K. I., \& DAVIS, C. (1984). Repetition priming and frequency attenuation in lexical access. Journal of Experimental Psychology: Learning, Memory, \& Cognition, 10, 680-698.

Forster, K. I., \& Shen, D. (1996). No enemies in the neighborhood: Absence of inhibitory neighborhood effects in lexical decision and semantic categorization. Journal of Experimental Psychology: Learning, Memory, \& Cognition, 22, 696-713.
Forster, K. I., \& VerEs, C. (1998). The prime lexicality effect: Formpriming as a function of prime awareness, lexical status, and discrimination difficulty. Journal of Experimental Psychology: Learning, Memory, \& Cognition, 24, 498-514.

GRAINGER, J., \& JACOBS, A. (1996). Orthographic processing in visual word recognition: A multiple read-out model. Psychological Review, 103, 518-565.

Grainger, J., O’Regan, J. K., Jacobs, A. M., \& Segui, J. (1989). On the role of competing word units in visual word recognition: The neighborhood frequency effect. Perception \& Psychophysics, $\mathbf{3}$, 189-195.

GRAINGER,J., \& SEgUI, J. (1990). Neighborhoodfrequency effects in visual word recognition: A comparison of lexical decision and masked identification latencies. Perception \& Psychophysics, 47, 191-198.

Johnson, N. F., \& Pugh, K. R. (1994). A cohort model of visual word recognition. Cognitive Psychology, 3, 240-346.

KUČERA, H., \& FRANCIS, W. N. (1967). Computationalanalysis of presentday American English. Providence, RI: Brown University Press.

McRae, K., \& Boisvert, S. (1998). Automatic semantic similarity priming. Journal of Experimental Psychology: Learning, Memory, \& Cognition, 24, 558-572.

Monsell, S., Doyle, C., \& Haggard, P. (1989). Effects of frequency on visual word recognition tasks: Where are they? Journal of Experimental Psychology: General, 118, 43-71.

NoRRIS, D. (1986). Word recognition: Context effects without priming. Cognition, 22, 93-136.

Paap, K., Newsome, S., McDonald, J., \& Schvaneveldt, R. (1982). An activation-verification model for letter and word recognition: The word superiority effect. Psychological Review, 89, 573-594.

Perea, M., \& Pollatsek, A. (1998). The effects of neighborhood frequency in reading and lexical decision. Journal of Experimental Psychology: Human Perception \& Performance, 24, 767-779.

Plaut, D. C. (1997). Structure and function in the lexical system: Insights from distributed models of word reading and lexical decision. Language \& Cognitive Processes, 12, 765-805.

Plaut, D. C., McClelland, J. L., Seidenberg, M. S., \& Patterson, K. (1996). Understanding normal and impaired word reading: Computational principles in quasi-regular domains. Psychological Review, 103, 56-115.

SeArs, C., Hino, Y., \& LuPKer, S. (1995). Neighborhood size and neighborhood frequency effects in word recognition. Journal of Experimental Psychology: Human Perception \& Performance, 21, 876-900.

SeArs, C., LupKer, S. J., \& Hino, Y. (1999). Orthographic neighborhood effects in perceptual identification and semantic categorization tasks: A test of the multiple read-out model. Perception \& Psychophysics, 61, 1537-1554.

Ziegler, J. C., \& Perry, C. (1998). No more problems in Coltheart's neighborhood: Resolving neighborhood conflicts in the lexical decision task. Cognition, 68, B53-B62.

\section{NOTES}

1. The results in each experiment were also analyzed using the more common procedure of excluding any RTs falling beyond $3 S D$ from the mean. For 11/13 means, the difference between the means calculated with the trimming procedure and the exclusion procedure was $5 \mathrm{msec}$ or less. In no case did the nature of any observed effect change (i.e., the patterns of differences remained the same), and the resulting $F$ ratios were virtually the same.

2. Recently, Ziegler and Perry (1998) showed that $\mathrm{N}$ is highly correlated with the number of neighbors that share the same body (body N). When body $\mathrm{N}$ is held constant, $\mathrm{N}$ has no effect on lexical decision times. 3. This proposal was suggested by Max Coltheart. 


\section{Items Used in Experiment 1}

\section{Nonwords}

$\mathrm{N}=1$ : braln craip broit norsh pavio ramin skide tarpot curton eriton fonate untode srobe $\mathrm{N}=2$ : doaly frope soims songe turve durate forver fatina wirmer straft carsel timud gorch $\mathrm{N}=3$ : tring shump shist prode poler froct insate walley frared sanned millet heawed roined

Nonexemplar Words

$\mathrm{N}=1$ : tutor quota berth coral floods manure piston yields banish shocks cracks placid kneel

$\mathrm{N}=2$ : marsh flakes spades chords vogue legion tenth impact retort repeal stony brisk blower

$\mathrm{N}=3+$ : hearth blots poster soles wastes medal fights blink rattle probe tease dearly needy

\section{Items Used in Experiment 2}

\section{Nonwords Based on Exemplars}

monse holse eigle whele speep tanda yebra bizard turple tarkey bobnat pogeon piraffe mooster naccoon kandaroo torboise elephint squirbel

Nonwords Based on Nonexemplars

creem mahor thugh nober kinse ptalm noole accest cishop tabric gonsip inpale bruiced groynds traipte ecstatin manpible parphlet cemplate

Low-Frequency Nonexemplar Words

feast grape spoil valve steal force tribe chrome hearth scrape throne dreamy acclaim bicycle deprive flannel diminish abnormal acquaint mystical

\section{High-Frequency Nonexemplar Words}

train smile clean drink fight learn teeth sought choose spread health slight balance concern kitchen patient argument distance identity separate

\section{Items Used in Experiment 4}

High-N Nonwords

chack swark goan duve fown boan ope bealer pij heg rav shunk pippy lomb koe

Low-N Nonwords

swen buson cadel frob eaple crol tanda toger roben barrot wolp furret yebra oyl tartle

Zero-N Nonwords

thrafe thowl repull pothe notrix touve fubade krewn daran jirce atova giyer tontry evad dagen

(Manuscript received October 23, 2000;

revision accepted for publication May 26, 2002.) 